PROCEEDINGS OF THE

AMERICAN MATHEMATICAL SOCIETY

Volume 125, Number 8, August 1997, Pages 2493-2500

S 0002-9939(97)04032-X

\title{
POINCARÉ FLOWS
}

\author{
SOL SCHWARTZMAN
}

(Communicated by Linda Keen)

\begin{abstract}
We study flows on a compact metric space $X$ with the property that corresponding to every non-zero element $\gamma$ of $H^{1}(X, Z)$ there is either a cross section associated with $\gamma$ or one associated with $-\gamma$. We obtain necessary and sufficient conditions for this to hold; on the $(k+1)$-dimensional torus these conditions take a classical form.
\end{abstract}

The present paper deals with cross sections of flows that take place on a compact metric space. For the time being it suffices to state that a cross section of such a compact dynamical system is a closed subset on which a homeomorphism that is intimately related to the flow is determined. For a flow on the torus a cross section must be a simple closed curve; consequently, theorems about homeomorphisms of the circle onto itself yield results about flows on the torus (see [13] for example).

Periodic orbits for the induced homeomorphism on a cross section correspond to periodic orbits of the original flow. This leads to theorems asserting the existence of periodic orbits for certain flows ([2], [11], [12]). Necessary and sufficient conditions for the existence of a cross section for a flow have been given by Fried, Fuller and the present author ([4], [7], [9], [12]).

However for compact manifolds of constant energy arising from a classical dynamical system, a theorem of Reeb asserts that no cross section can exist. Instead, a generalization known as a surface of section can sometimes be found. These were first considered by Poincaré, who showed using them that periodic orbits could be shown to exist in the restricted three-body problem if one could prove that an area-preserving homeomorphism of an annulus that rotated the bounding circles in opposite senses had a fixed point. This conjecture was proved by G. D. Birkhoff, who applied it to get periodic orbits for other flows [2]. Recently J. Franks proved that an area-preserving homeomorphism of an annulus that possessed at least one periodic point possesses infinitely many [3]. Using a surface of section he showed that this, augmented by a result of V. Bangert [1], sufficed to show that there are infinitely many closed geodesics on a Riemannian two-sphere.

In Theorem $\mathrm{M}$ of [4] Fried gives a necessary and sufficient condition for the existence of a surface of section. However the present author must confess that he was unable to understand the proof of this result.

Going back to cross sections as originally discussed, we will indicate below how one associates a one-dimensional cohomology class of $X$ with each cross section of a flow on $X$. Two cross sections of the same flow corresponding to the same

Received by the editors February 26, 1996.

1991 Mathematics Subject Classification. Primary 58F25.

(C)1997 American Mathematical Society 
cohomology class can be mapped homeomorphically onto each other in such a way that the homeomorphisms induced on the cross sections by the flow correspond. If $\left(K_{1}, \varphi_{1}\right)$ and $\left(K_{2}, \varphi_{2}\right)$ are discrete dynamical systems arising as cross sections to the same flow, then these systems are said to be flow equivalent [5].

In Theorem A of [5] Fried shows that under certain circumstances one can single out a finite number of cohomology classes of a flow, each of which correspond to a cross section. He then gives some properties characterizing the resulting finite collection of discrete dynamical systems.

It is easy to show that it is never the case that a cohomology class $\gamma$ and its negative $-\gamma$ both correspond to a cross section.

On the torus however smooth topologically transitive flows without stationary points have the property that for every non-zero element $\gamma$ of $H^{1}\left(T^{2}, Z\right)$ either there is a cross section corresponding to $\gamma$ or there is one corresponding to $-\gamma$. We call such flows on an arbitrary compact manifold Poincaré flows and study them in this paper.

Before proceeding to the main body of this paper it is worthwhile to note that if one considers ergodic measure-preserving flows on a standard measure space (without any topology), the measure theory analogue of a cross section always exists. One can also consider the measure-theoretic analogue of flow equivalence. This was first studied by Kakutani and is called Kakutani equivalence of ergodic actions. It was originally conjectured that there was only one Kakutani equivalence class; this is now known to be false. Although the present author has not seen a copy, a review in the Bulletin of the A.M.S. indicates that [8] should be a good reference for this material.

We are going to apply results about asymptotic cycles [9] to the study of a special class of flows. To start out with, let us consider differential equations

$$
\frac{d x_{i}}{d t}=X_{i}\left(x_{1}, \ldots, x_{k}, t\right)
$$

where the functions $X_{i}$ are of class $C^{1}$ and periodic of period one in each of their variables, including $t$. We can take these equations as defining a flow on the $(k+1)$ dimensional torus. We are going to prove that statements A), B), and C) below are equivalent.

A) There exist constants $\lambda_{1}, \ldots, \lambda_{k}$ such that:

(1) The numbers $\left\{1, \lambda_{1}, \ldots, \lambda_{k}\right\}$ form a rationally independent set.

(2) $\lim _{t \rightarrow \infty} \frac{x_{i}(t)}{t}=\lambda_{i}$ for each solution $\left\{x_{1}(t), \ldots, x_{k}(t)\right\}$ to our differential equations.

When $k=1$, condition A) just tells us that the flow we get on the twodimensional torus has an irrational rotation number.

B) For any non-trivial set of integers $\left\{n_{0}, n_{1}, \ldots, n_{k}\right\}$ it is either true that for every solution $\left\{x_{1}(t), \ldots, x_{k}(t)\right\}, \lim _{t \rightarrow \infty}\left(n_{0} t+n_{1} x_{1}(t)+\cdots+n_{k} x_{k}(t)\right)$ is $+\infty$, or that for every solution this limit is $-\infty$.

C) For every non-zero one-dimensional cohomology class $\gamma$, either there is a global cross section associated with $\gamma$ or there is a global cross section associated with $-\gamma$.

We will recall below what a global cross section is and how we associate a onedimensional cohomology class with it. For now we simply note that it never happens, for a one-dimensional cohomology class $\gamma$, that we can get a cross section associated with $\gamma$ and that we can also get a cross section associated with $-\gamma$. 
Actually it is going to turn out that we can reformulate statements A), B), and C) so that they make sense and are equivalent for flows in any compact metric space $X$, provided that the natural map of the one-dimensional Cech cohomology group into the singular cohomology group is an isomorphism and the first Betti number of $X$ is finite. Of course these restrictions hold if $X$ is any compact manifold. (It is necessary to place these restrictions in order to make the results in [9] hold. The results in [9] were stated for an arbitrary compact metric space but this was unduly optimistic.)

We will proceed to the proof that these statements are equivalent.

A) $\rightarrow$ B): Let $\left\{n_{0}, n_{1}, \ldots, n_{k}\right\}$ be any non-trivial set of integers. Then

$$
\begin{aligned}
n_{0} t+n_{1} x_{1}(t)+\cdots+n_{k} x_{k}(t) & =n_{0} t+n_{1}\left(\lambda_{1} t+o(t)\right)+\cdots+n_{k}\left(\lambda_{k} t+o(t)\right) \\
& =\left(n_{0}+n_{1} \lambda+\cdots+n_{k} \lambda_{k}\right) t+o(t) .
\end{aligned}
$$

Since $\left\{1, \lambda_{1}, \lambda_{2}, \ldots, \lambda_{k}\right\}$ are rationally independent, $n_{0}+n_{1} \lambda_{1}+\cdots+n_{k} \lambda_{k}$ is not zero. If it is positive, $\lim _{t \rightarrow \infty} n_{0} t+\cdots+n_{k} x_{k}(t)$ is $+\infty$, otherwise it is $-\infty$.

Before proving that $\mathrm{B}$ ) implies $\mathrm{C}$ ), we recall that when we have a global cross section of a flow on a compact metric space $X$ we have a compact subspace $K$, a homeomorphism $\varphi$ of $K$ onto itself and a continuous, everywhere positive function $F$ on $K$. In addition, if $\Omega$ is the subset of $K \times R$ consisting of all $(x, t)$ with $0 \leq t \leq F(x)$, we must have a map $H$ of $\Omega$ onto $X$ such that:

a) $H(x, 0)=H\left(\varphi^{-1}(x), F\left(\varphi^{-1}(x)\right)\right)=x$.

b) If, for $q \in X, q t$ denotes the point into which $q$ is sent by the flow after time $t$, we must have $H(x, t)=(H(x, 0)) t$ for all $0 \leq t \leq F(x)$.

c) For each $x \in K$, the map $H$ identifies $(x, 0)$ with $\left(\varphi^{-1}(x), F\left(\varphi^{-1}(x)\right)\right)$ and no other identifications occur.

The subspace $K$ is called the global cross section and $\varphi$ is called the return map.

Given this setup, we define a real-valued function $\Theta(x, t)$ on $\Omega$ by letting $\Theta(x, 0)$ $=0$ and $\Theta(x, F(x))=2 \pi$ for all $x \in K$, and extending $\Theta$ linearly from $(x, 0)$ to $(x, F(x))$. Then $f(H(x, t))=e^{i \Theta(x, t)}$ for one and only one continuous complexvalued function $f$ of absolute value one on $X$.

Next we recall that if $C(X)$ is the collection of all continuous complex-valued functions of absolute value one on $X$, made into a group under pointwise multiplication, and $R(x)$ is the subgroup consisting of all elements $g(x) \in C(X)$ which can be written in the form $e^{i \alpha(x)}$, where $\alpha(x)$ is a continuous real-valued function, then the quotient group $C(X) / R(X)$ is naturally isomorphic to the one-dimensional Cech cohomology group of $X$ with integer coefficients. Since we have associated with each cross section of the flow on $X$ a continuous complex-valued function $f$ of absolute value one, the element $\bar{f} \in C(X) / R(X)$ gives us a one-dimensional cohomology class associated with the cross section.

This is an important invariant associated with a cross section; for example, if $X$ is a compact manifold and $K_{1}$ and $K_{2}$ are two cross sections of the same flow with corresponding return maps $\varphi_{1}$ and $\varphi_{2}$, then if both cross sections correspond to the same cohomology class there is a homeomorphism from $K_{1}$ to $K_{2}$ that makes $\varphi_{1}$ correspond to $\varphi_{2}$ [10]. This need not be true if $K_{1}$ and $K_{2}$ correspond to different cohomology classes.

Next we are going to introduce some notation that arises when we have a continuous function $f$ of absolute value one on a compact metric space $X$ where we have a flow. If $p \in X$, there exists a continuous real function $\Theta(t)$ such that $f(p t)=e^{i \Theta(t)}$. 
Since $\Theta(t)$ is unique up to an additive constant, for any $T, \Theta(T)-\Theta(0)$ is uniquely defined. We denote this quantity by $\Delta \arg f_{(p, p T)}$. A theorem of Fuller [7] asserts that if $h$ is a continuous complex-valued function of absolute value one on $X$ and $\bar{h}$ is the corresponding one-dimensional cohomology class, a necessary and sufficient condition that there exists a cross section with which $\bar{h}$ is associated is that for every $p \in X$ there exists a $T>0$ such that $\Delta \arg h_{(p, p T)}$ is greater than zero. It is easy to see that if such a $T$ can be found for every $p \in X$, then a $T$ independent of $p$ can be found. Now we can proceed to the next step in our proof.

$\mathrm{B}) \rightarrow \mathrm{C})$ : Every one-dimensional cohomology class in $C(X) / R(X)$, where $X$ is the $(k+1)$-dimensional torus, contains a function of the form $e^{2 \pi i\left(n_{0} t+n_{1} x_{1}+\cdots+n_{k} x_{k}\right)}$. If $p=\left(t_{0}, x_{1}, \ldots, x_{k}\right)$ and $\left(x_{1}(t), \ldots, x_{k}(t)\right)$ is the solution to our differential equations such that $x_{i}\left(t_{0}\right)=x_{i}$, then

$$
\begin{aligned}
& \Delta \arg \left(e^{2 \pi i\left(n_{0} t+n_{1} x_{1}+\cdots+n_{k} x_{k}\right)}\right)_{(p, p T)} \\
& \quad=2 \pi\left(n_{0} T+n_{1}\left(x_{1}\left(T+t_{0}\right)-x_{1}\left(t_{0}\right)\right)+\cdots+n_{k}\left(x_{k}\left(T+t_{0}\right)-x_{k}\left(t_{0}\right)\right) .\right.
\end{aligned}
$$

By B) it is either true that for all $p$ this goes to $+\infty$ or for all $p$ this goes to $-\infty$. By Fuller's Theorem in the first case there is a cross section corresponding to our original cohomology class and in the second case there is a cross section corresponding to the negative of our cohomology class. (Of course, we are assuming that $\left(n_{0}, n_{1}, \ldots, n_{k}\right)$ is not trivial.)

Next we want to prove that C) implies A). To do this we will need another necessary and sufficient condition for a cross section to exist that is associated with a given cohomology class $\bar{h}$. To state this condition we will need the notion of an asymptotic cycle, which was introduced in [9].

For simplicity we will assume that our space $X$ is a smooth compact manifold. Suppose we are given a continuous function $f$ from $[0, \infty)$ into $X$. Put a Riemannian metric on $X$ and for each $T>0$ choose a path in $X$ from $f(T)$ to $f(0)$ having length less than or equal to the diameter of $X$. Let $C_{T}$ be the closed curve obtained by closing up the path given by $f$ restricted to $[0, T]$ by this path, and let $\bar{C}_{T}$ be the element of the one-dimensional homology group of $X$ with real coefficients determined by $C_{T}$.

If $\lim _{T \rightarrow \infty} \frac{1}{T} \bar{C}_{T}$ exists, then it also exists and has the same value if we use any other Riemannian metric and close up by other paths. This is so because $\lim _{T \rightarrow \infty} \frac{1}{T} \bar{C}_{T}$ exists if and only if $\lim _{T \rightarrow \infty} \frac{1}{T} \Delta \arg h_{(p, p T)}$ exists for each $h \in C(X)$ and the latter limits determine $\lim _{T \rightarrow \infty} \frac{1}{T} \bar{C}_{T}$. We call this the asymptotic cycle determined by $f$. If we have a continuous flow in $X$ and $p \in X$ is any quasi-regular point, and if we let $f(t)=p t$, then there is an asymptotic cycle associated with $f$. We denote this by $A_{p}$ and call it the asymptotic cycle determined by $p$. (A point $p$ is called quasi-regular provided, for every continuous real-valued function $h$ defined in $X, \lim _{T \rightarrow \infty} \frac{1}{T} \int_{0}^{T} h(k t) d t$ exists. The set of quasi-regular points has measure one with respect to every invariant probability measure [14].) If $h$ is a continuous complex-valued function of absolute value one on $X$ and $\bar{h}$ is the corresponding element of $H^{1}(X, Z)$, the coefficient homomorphism of $H^{1}(X, Z)$ into $H^{1}(X, R)$ gives us an element of $H^{1}(X, R)$ associated with $\bar{h}$. This determines a linear functional on $H_{1}(X, R)$. For any quasi-regular point $p$ we denote the value of the linear functional on $A_{p}$ by $A_{p}(\bar{h})$. Of course, for fixed $p$, the map sending $\bar{h}$ into $A_{p}(\bar{h})$ is a homomorphism of $H^{1}(X, Z)$ into $R$. Moreover $A_{p}(\bar{h})=$ $\lim _{T \rightarrow \infty} \frac{1}{2 \pi T} \Delta \arg h_{(p, p T)}$. 
Now we are ready to proceed with the proof.

For any invariant probability measure $\mu$ we may define $A_{\mu}=\int A_{p} d \mu(p)$. This makes sense since $A_{p}$ is an element of a finite-dimensional vector space over the reals. Interpreting $A_{\mu}(\bar{h})$ in the obvious way we get $A_{\mu}(\bar{h})=\int A_{p}(\bar{h}) d \mu(p)$. Since the quasi-regular points $p$ are of $\mu$-measure one, if $A_{p}(\bar{h})>0$ for each quasi-regular point $p$, then $A_{\mu}(\bar{h})>0$ for each invariant probability measure $\mu$. In [9] it is proven that there is a cross section associated with $\bar{h}$ if and only if $A_{\mu}(\bar{h})>0$ for every invariant probability measure $\mu$. Thus there is a cross section associated with $\bar{h}$ if and only if $A_{p}(\bar{h})>0$ for every quasi-regular point $p$.

$\mathrm{C}) \rightarrow \mathrm{A})$ : Let $X$ be our $(k+1)$-dimensional torus. For any quasi-regular point $p$, $A_{p}(\bar{h})$ is positive if $\bar{h}$ corresponds to a cross section and negative otherwise (unless $\left.n_{0}=n_{1}=\cdots=n_{k}=0\right)$. Thus the homomorphism of $C(X) / R(X)$ into the real line sending $\bar{h}$ into $A_{p}(\bar{h})$ is injective.

Now, if $p_{1}$ and $p_{2}$ are two quasi-regular points, by virtue of the isomorphisms of $C(X) / R(X)$ with its images under the homomorphisms determined by $A_{p_{1}}$ and $A_{p_{2}}$ we get an isomorphism of these images. Since $A_{p_{1}}(\bar{h})$ and $A_{p_{2}}(\bar{h})$ are positive real numbers exactly when $\bar{h}$ corresponds to a cross section, this isomorphism is order preserving. This is the crucial point in our proof. In fact, if we have an order-preserving isomorphism of one additive subgroup of the real line into another this extends to an order-preserving isomorphism of the real line onto itself, that is, to a map sending $x$ into $\lambda x$ where $\lambda$ is some positive constant. This is trivial when our subgroups are infinite cyclic; in the remaining case both subgroups are dense in the real line and the additive group of the real line can be regarded as the completion of each of these subgroups in the order topology. Thus our orderpreserving isomorphism of subgroups extends to an order-preserving isomorphism of their completions.

We therefore see that for our two quasi-regular points $p_{1}$ and $p_{2}$ there is a positive constant $\lambda$ so that for any $\bar{h} \in C(X) / R(X), A_{p_{2}}(\bar{h})=\lambda A_{p_{1}}(\bar{h})$. Therefore, $A_{p_{1}}$ and $A_{p_{2}}$ determine linearly dependent linear functionals on $H^{1}(X, R)$, from which it follows that $A_{p_{1}}$ and $A_{p_{2}}$ are themselves linearly dependent. Therefore, there exists a one-dimensional subspace of $H_{1}(X, R)$ which contains $A_{p}$ for every quasi-regular point $p$.

Let $h$ equal $e^{2 \pi i t}$. Then for any quasi-regular point $p$,

$$
A_{p}(\bar{h})=\lim _{T \rightarrow \infty} \frac{1}{2 \pi T} \Delta \arg h_{(p, p T)}=1
$$

Therefore, $A_{p}$ is the same for all quasi-regular points $p$. Now it is a fact that if $A_{p}$ is the same for all quasi-regular points $p$, then the asymptotic cycle $A_{q}$ is defined and has the same constant value for all points $q \in X$. (If one introduces asymptotic cycles as in [9], this follows from the fact that if $f$ is any continuous function $X$ such that the value of $\lim _{T \rightarrow \infty} \frac{1}{T} \int_{0}^{T} f(p t) d t$ is the same as $T \rightarrow \infty$ for all quasi-regular points $p$, then this limit exists and has the same value for all points $p$.)

Next, let $A$ denote the common value of the asymptotic cycles $A_{p}$. Let $h_{0}=e^{2 \pi i t}$ and $h_{i}=e^{2 \pi i x_{i}}(1 \leq i \leq k)$. Then $\bar{h}_{0}, \ldots, \bar{h}_{k}$ yields a basis for $H^{1}(X, R)$. Take the dual basis for $H_{1}(X, R)$ and let the components of $A$ with respect to this dual basis be $\left(1, \lambda_{1}, \ldots, \lambda_{k}\right)$. (We have already seen that the zeroth component of $A$ equals 1.) Then if $\left(x_{1}(t), \ldots, x_{k}(t)\right)$ is any solution, and we let $p \in X$ correspond 
to $\left(0, x_{1}(0), \ldots, x_{k}(0)\right)$

$$
A\left(\bar{h}_{i}\right)=\lambda_{i}=\lim _{T \rightarrow \infty} \frac{1}{2 \pi T} \Delta\left(\arg h_{i}\right)_{(p, p T)}=\lim _{T \rightarrow \infty} \frac{x_{i}(T)-x_{i}(0)}{T}=\lim \frac{x_{i}(T)}{T}
$$

for $1 \leq i \leq k$.

Finally, suppose $\left(n_{0}, n_{1}, \ldots, n_{k}\right)$ is a non-trivial set of integers and let $h=$ $e^{2 \pi i\left(n_{0} t+n_{1} x_{1}+\cdots+n_{k} x_{k}\right)}$. Then $A(\bar{h})=n_{0}+n_{1} \lambda_{1}+\cdots+n_{k} \lambda_{k}$. But either $\bar{h}$ or $-\bar{h}$ corresponds to a cross section so $n_{0}+n_{1} \lambda_{1}+\cdots+n_{k} \lambda_{k} \neq 0$. Thus $\left\{1, \lambda_{1}, \ldots, \lambda_{k}\right\}$ is a rationally independent set of numbers. This concludes the proof that A), B), and $\mathrm{C}$ ) are equivalent.

As stated in the introduction our result generalizes to the case where $X$ is a compact metric space with finite first Betti number such that the natural map of the one-dimensional Čech cohomology group with integer coefficients into the onedimensional singular group is an isomorphism. In the case just treated, where $X$ is the $(k+1)$-dimensional torus, we could have used the functions $e^{2 \pi i t}, e^{2 \pi i x_{1}}, \ldots$, $e^{2 \pi i x_{k}}$ instead of $t, x_{1}, \ldots x_{k}$ to state our conditions. For example, $\lim _{t \rightarrow \infty} \frac{x_{i}(t)}{t}$ is the same as $\lim _{t \rightarrow \infty} \frac{1}{2 \pi t} \Delta \arg e_{(p, p t)}^{2 \pi i x_{i}}$.

We therefore assume, in the general case, that we have continuous functions $h_{0}, h_{1}, \ldots, h_{k}$ yielding a basis for $C(X) / R(X)$. In order that $h_{0}$ correspond to $e^{2 \pi i t}$ we assume that $h_{0}$ satisfies $h_{0}(p t)=e^{2 \pi i t} h_{0}(p)$; in other words, that $h_{0}$ is an eigenfunction with eigenvalue $2 \pi$. Then, exactly as above, we can prove that the following conditions are equivalent:

$\left.\mathrm{A}_{0}\right)$ There exist constants $\lambda_{1}, \ldots, \lambda_{k}$ such that:

a) $\left\{1, \lambda_{1}, \ldots, \lambda_{k}\right\}$ are rationally independent.

b) $\lim _{t \rightarrow \infty} \frac{\left(\Delta \arg h_{i}\right)_{(p, p t)}}{2 \pi t}=\lambda_{i}(1 \leq i \leq k)$ for all $p$.

$\left.\mathrm{B}_{0}\right)$ For any non-trivial set of integers $n_{0}, n_{1}, \ldots, n_{k}$, either $\lim _{t \rightarrow \infty} n_{0} t+$ $\frac{n_{1} \Delta\left(\arg h_{1}\right)_{(p, p t)}}{2 \pi}+\cdots+\frac{n_{k} \Delta\left(\arg h_{k}\right)_{(p, p t)}}{2 \pi}=+\infty$ for every $p \in X$, or this limit is $-\infty$ for every $p \in X$.

$\mathrm{C}_{0}$ ) For every non-zero element $\gamma$ of $C(X) / R(X)$ either there is a cross section associated with $\gamma$ or there is a cross section associated with $-\gamma$.

We are now ready to give the final form of our results.

Let $X$ be a compact metric space with finite first Betti number such that the natural map of the one-dimensional Čech cohomology group with integer coefficients into the one-dimensional singular group is an isomorphism. Let $h_{0}, h_{1}, \ldots, h_{k}$ be continuous functions of absolute value one on $X$ that yield a basis for $C(X) / R(X)$.

Assume we have a flow on $X$. Let $\Delta \ell_{(p, p T)}$ equal $\sqrt{\sum_{i=0}^{k}\left(\Delta \arg h_{(p, p T)}\right)^{2}}$.

Theorem. The following sets of statements are equivalent:

$\mathrm{A}_{1}$ ) a) $\lim _{T \rightarrow \infty} \Delta \ell_{(p, p T)}=\infty$ for each $p \in X$.

b) There exist rationally independent constants $\mu_{0}, \mu_{1}, \ldots, \mu_{k}$ such that for each $p \in X$ and $0 \leq i \leq k$ we have $\lim _{T \rightarrow \infty} \frac{\Delta \arg h_{i(p, p T)}}{\Delta \ell_{(p, p T)}}=\mu_{i}$.

$\left.\mathrm{B}_{1}\right)$ For every non-trivial set of integers $n_{0}, \ldots, n_{k}$ either

$$
\lim _{T \rightarrow \infty} \sum_{i=0}^{k} n_{i} \Delta \arg h_{i_{(p, p T)}}=\infty
$$


for every $p \in X$, or

$$
\lim _{T \rightarrow \infty} \sum_{i=0}^{k} n_{i} \Delta \arg h_{i_{(p, p T)}}=-\infty
$$

for every $p \in X$.

$\mathrm{C}_{1}$ ) For every non-zero element $\gamma \in C(X) / R(X)$ either there is a cross section associated with $\gamma$ or there is one associated with $-\gamma$.

Proof. $\left.\mathrm{A}_{1}\right) \rightarrow \mathrm{B}_{1}$ ): This follows by essentially the same argument we used previously to show A) $\rightarrow$ B).

$\left.\mathrm{B}_{1}\right) \rightarrow \mathrm{C}_{1}$ ): Same argument as that which showed $\left.\mathrm{B}\right) \rightarrow \mathrm{C}$ ).

$\left.\mathrm{C}_{1}\right) \rightarrow \mathrm{A}_{1}$ ): First we note that it is sufficient merely to show that $\mathrm{C}_{1}$ ) implies there exist functions $\bar{h}_{0}, \ldots, \bar{h}_{k}$ yielding the same eigenvalue classes as $h_{0}, \ldots, h_{k}$ for which the statements analogous to a) and b) of $\mathrm{A}_{1}$ ) hold. This is so because $\left|\Delta \arg \bar{h}_{i_{(p, p T)}}-\Delta \arg h_{i_{(p, p T)}}\right|$ is uniformly bounded for all $p$ and $T$.

Next we observe that it is sufficient to prove, given $\mathrm{C}_{1}$ ), that $\mathrm{A}_{1}$ ) holds for a modification of our original flow that consists of altering the rate at which orbits are traversed but retains the same orbits.

Making use of the possibility of making both these modifications we see that since there certainly is a cross section corresponding to the element of $C(X) / R(X)$ determined by $h_{0}$ we may assume that for all $p$ and $T, h_{0}(p T)=e^{2 \pi i T} h_{0}(p)$. Thus we are in a situation where $\mathrm{C}_{0}$ ) of our previous discussion holds and we know that $\mathrm{C}_{0}$ ) implies $\mathrm{A}_{0}$ ).

Clearly for the functions $h_{0}, \ldots, h_{k}$ we are now dealing with, $\lim _{T \rightarrow \infty} \Delta \ell_{(p, p T)}=$ $\infty$. Moreover

$$
\lim _{T \rightarrow \infty} \frac{\Delta \ell_{(p, p T)}}{2 \pi T}=\lim _{T \rightarrow \infty} \sqrt{1+\sum_{i=1}^{k} \frac{\left(\Delta \arg h_{i_{(p, p T)}}\right)^{2}}{(2 \pi T)^{2}}}=\sqrt{1+\sum_{i=1}^{k} \lambda_{i}^{2}}
$$

where the $\lambda_{i}$ are as in $\mathrm{A}_{0}$ ). Then, for $1 \leq i \leq k$,

$$
\lim _{T \rightarrow \infty} \frac{\Delta \arg h_{i_{(p, p T)}}}{\Delta \ell_{(p, p T)}}=\frac{\lambda_{i}}{\sqrt{1+\sum_{i=1}^{k} \lambda_{i}^{2}}} .
$$

Also

$$
\lim _{T \rightarrow \infty} \frac{\Delta \arg h_{0_{(p, p T)}}}{\Delta \ell_{(p, p T)}}=\frac{1}{\sqrt{1+\sum_{i=1}^{k} \lambda_{i}^{2}}}
$$

Thus $\mathrm{A}_{1}$ ) holds.

The following will prove our final result. Suppose on the $n$-dimensional torus we consider a flow obtained from differential equations $\frac{d x}{d t}=X_{i}\left(x_{1}, \ldots, x_{n}\right)$ where the $X_{i}\left(x_{1}, \ldots, x_{n}\right)$ are $C^{1}$ functions of period one in each of the variables $x_{1}, \ldots, x_{n}$. Then it follows easily that the following are equivalent:

$\left.\mathrm{A}^{1}\right)$ 1) If $\ell(t)=\sqrt{\left(\sum x_{i}(t)\right)^{2}}$, then for any solution to our differential equation $\lim _{t \rightarrow \infty} \ell(t)=\infty$.

2) There exist rationally independent numbers $\mu_{1}, \ldots, \mu_{n}$ such that if $x_{1}(t), \ldots$, $x_{n}(t)$ is any solution to our differential equations, then $\lim _{t \rightarrow \infty} \frac{x_{i}(t)}{\ell(t)}=\mu_{i}$.

$\left.\mathrm{B}^{1}\right)$ If $k_{1}, \ldots, k_{n}$ is any nontrivial set of integers, then it is either true that $\lim _{t \rightarrow \infty} k_{1} x_{1}(t)+\cdots+k_{n} x_{n}(t)=+\infty$ for every solution $x_{1}(t), \ldots, x_{n}(t)$ to our differential equations, or this limit is $-\infty$ for every solution. 
$\mathrm{C}^{1}$ ) For each non-zero $\gamma \in C(X) / R(X)$ either there exists a cross section corresponding to $\gamma$ or there exists one corresponding to $-\gamma$.

\section{REFERENCES}

1. V. Bangert, On the existence of closed geodesics on trio-spheres, International Journal of Math. 4 No. 1 (1993), 1-10. MR 94d:58036

2. G. D. Birkhoff, Dynamical Systems, A.M.S. Colloquium Publications, Vol. 9. MR 35:1

3. J. Franks, Geodesics on $S^{2}$ and periodic points of annulus homeomorphisms, Inventiones Math. Vol. 108 Fasc. 2 (1992), 403-418. MR 93f:58192

4. David Fried, The geometry of cross sections to flows, Topology 21 (1982), 353-357. MR 84d:58068

5. David Fried, Flow equivalence, hyperbolic systems and a new zeta function for flows, Comment. Math. Helv. 57 (1982), 237-259. MR 84g:58083

6. F. Brock Fuller, The existence of periodic points, Annals of Math. 2757 (1953), 229-230. MR 14:669f

7. F. Brock Fuller, On the surface of section and periodic trajectories, Amer. Journal of Math. 87 (1965), 473-480. MR 31:3680

8. M. G. Nadkami, Basic Ergodic Theory, Texts and Readings in Math. Vol. 6, Hindustan Book Agency, 1995.

9. S. Schwartzman, Asymptotic cycles, Annals of Math. 66 No. 2 (1957), 270-284. MR 19:568i

10. S. Schwartzman, Global cross sections of compact dynamical systems, Proc. Nat. Acad. Sci. 48 No. 5 (1962), 786-791. MR 25:1543

11. S. Schwartzman, Parallel vector fields and periodic orbits, Proc. A.M.S. 44 No. 1, 167-168. MR 48:9767

12. S. Schwartzman, Periodic orbits for generalized gradient flows, Canadian Math. Bull 38 (1) (1995), 117-119. MR 96b:57031

13. C. L. Siegel, Note on differential equations on the torus, Annals of Math. 46 (1945). MR 7:117g

14. J. C. Oxtoby, Ergodic sets, Bull Amer. Math. Soc. 58 (1952), 116. MR 13:850e

Department of Mathematics, University of Rhode Island, Kingston, Rhode Island 02881-0806 